\title{
Use of the Symmetries in the Study of Vibration Response of a Hollow Cylinder
}

\author{
Călin Itu ${ }^{1}$, Sorin Vlase ${ }^{1,2, * \mathbb{D}}$, Marin Marin ${ }^{3} \mathbb{D}$ and Ana Toderiță ${ }^{2}$ \\ 1 Department of Mechanical Engineering, Transilvania University of Brașov, B-dul Eroilor 20, \\ 500036 Brașov, Romania; calinitu@unitbv.ro \\ 2 Romanian Academy of Technical Sciences, B-dul Dacia 26, 030167 Bucharest, Romania; ana.toderita@unitbv.ro \\ 3 Department of Mathematics, Transilvania University of Brașov, B-dul Eroilor 20, 500036 Brașov, Romania; \\ m.marin@unitbv.ro \\ * Correspondence: svlase@unitbv.ro; Tel.: +40-722-643-020
}

check for updates

Citation: Itu, C.; Vlase, S.; Marin, M. Toderiță, A. Use of the Symmetries in the Study of Vibration Response of a Hollow Cylinder. Symmetry 2021, 13, 2145. https://doi.org/10.3390/ sym13112145

Academic Editors: Raffaele Barretta and Sergei Alexandrov

Received: 2 October 2021

Accepted: 8 November 2021

Published: 10 November 2021

Publisher's Note: MDPI stays neutral with regard to jurisdictional claims in published maps and institutional affiliations.

Copyright: (c) 2021 by the authors. Licensee MDPI, Basel, Switzerland. This article is an open access article distributed under the terms and conditions of the Creative Commons Attribution (CC BY) license (https:/ / creativecommons.org/licenses/by/ $4.0 /)$.

\begin{abstract}
The paper studies the vibration response of an elastic solid that has geometric symmetries. These determine special properties of the equations of motion of such a system, presented in the case of a cylindrical body (hollow cylinder). The properties of the eigenvalues and eigenmodes of these systems are theoretically established. A validation of these results is made using the finite element method. The use of the obtained results can lead to an easing of the vibration analysis of such a system and, consequently, to the decrease of the cost related to the design and manufacture of such a structure. The properties presented and demonstrated in the paper can simplify the numerical calculation and experimental verifications of such a structure. Serving these symmetries, the computation cost decrease substantially and will depend not in the number of the identical parts.
\end{abstract}

Keywords: symmetry; hollow cylinder; FEM; eigenvalue; eigenmode; vibration

\section{Introduction}

A problem that often arises in the field of civil engineering, thus gaining importance, is the use of symmetries that can be solved by obtaining the ease of calculation and design time of a structure with identical parts. In engineering practice, most buildings, works of art, halls, etc., are composed of identical parts and have symmetries. This has been happening since antiquity and the reasons are many. First, a lighter, faster design, then a cheaper realization and, less important for engineers but important for beneficiaries, for aesthetic reasons. In engineering, civil engineering and other fields, such as the machine or equipment manufacturing industry, the automotive industry, or the aerospace industry, there are products, elements, machines and components that contain identical, repetitive parts, which have in their composition parts that have symmetries of different types. Within the constructions, most of the buildings, works of art, halls, etc., are composed of identical parts and have symmetries. These properties can be used successfully to facilitate the static and dynamic analysis of some structures. Symmetries of different types that provide specific properties have been observed for a long time and are used especially in the static case.

Structures that present different symmetries or structures with repetitive elements are common both in the field of engineering applications, architecture and construction, but also in nature. These mechanical systems have identical geometry, properties, boundary conditions and connection with the main structure.

As a general result the eigenvalues and eigenmodes for a mechanical system are defined by the composition, geometry and properties of the whole system. For structures with symmetries or/and repetitive parts, some eigenvalues and eigenmodes are identical to those of the whole system and this can facilitate the calculation of such a system. In this 
way the necessary calculation time and costs for the design of such a structure decreases considerably.

From the multitude of aspects that can be studied we focused on the analysis of the dynamic response of these structures, especially on the study of vibration behavior. Although for the static case the problem has been studied and properties have been deduced that facilitate the design and calculation work, in the field of dynamic analysis the use of these properties is still reduced. In the paper, we propose to identify some vibration properties of cylindrical bodies, properties that can make the process of design, calculation and manufacturing of such structures easier.

In engineering practice (but also in the natural world) there are many structures presenting some type of symmetry [1]. A structure assembled by a group of identical substructures can be defined as repetitive. These identical substructures must be identical in all the parameters defining the mechanical behavior (geometrical shape, physical properties, boundary conditions and connections). To make a vibrational analysis of such types of structure, using finite element method (FEM), is necessary to mesh the whole structure, a very costly step. The use of the information about the symmetry properties of such structures could lead to a more economical way to obtain the results. In this way, for example, the eigenvalues of the whole structure can be determined from those of its single substructure. The properties of the repetitive structure were studied in [2-8]. Another way to study this type of problem is to analyze the wave propagations in linear periodic systems [9-13].

Thus far, the work presented previously has focused on the study of discrete systems. In the paper, we extend the method to continuous mechanical systems. The developed models are solved using the finite element method, which possesses, the well-developed methods for determining the eigenfrequencies and the eigenmodes. This can become a major advantage using the classical finite elements for the study of vibrations of large structures. Conventional FEM is used in the paper to form the classical mass and stiffness matrices.

There are many kinds of symmetry. In this paper, we will study a hollow cylinder that presents a mirror-symmetry and an axis-symmetry. A mirror-symmetric structure is if its geometric shape, physical properties and boundary conditions are all symmetric with respect to a plane (or a straight line). An axis-symmetric structure is if its geometry, physical properties and boundary conditions are all unaltered after rotating it by an arbitrary angle with respect to an axis (a straight line).

The existence of symmetries can be used to facilitate the analysis of vibration of bodies or structures. Although properties concerning the vibrations behavior of the mechanical systems with certain symmetries were set by many researchers $[14,15]$ and some are known intuitively, after practice, by the users of finite element software, a systematic study of the effects of these symmetries is not yet done. The problem is complex, it is necessary to study several types of symmetries and their effect on the behavior of bodies or mechanical vibration systems. Meirovitch [6] specified, allusively, the importance of considering the symmetries. A first systematic approach to the problem is made in [10] and developed in [16]. A rigorous mathematical demonstration of mechanical properties of the equations of motion written for symmetrical systems with symmetries is made in [17]. New researches in the domain are presented in [18-27]. For the problems with complex symmetries a systematic study is not yet done.

\section{Materials and Methods}

In the following, we will present the motion equations for a cylinder considered as a continuous solid [28-30]. Motion equations can be written, using the cylindrical coordinates $(r, \theta, z)$, in terms of stress components $\sigma_{r}, \sigma_{\theta}, \sigma_{z}, \sigma_{r \theta}, \sigma_{r z}, \sigma_{\theta z}$ are expressed by:

$$
\frac{\partial \sigma_{r}}{\partial r}+\frac{1}{r} \frac{\partial \sigma_{r \theta}}{\partial \theta}+\frac{\partial \sigma_{r z}}{\partial z}+\frac{1}{r}\left(\sigma_{r}-\sigma_{\theta}\right)+b_{r}=\mu \ddot{u}_{r},
$$




$$
\begin{aligned}
& \frac{\partial \sigma_{r \theta}}{\partial r}+\frac{1}{r} \frac{\partial \sigma_{\theta}}{\partial \theta}+\frac{\partial \sigma_{\theta z}}{\partial z}+\frac{2}{r} \sigma_{r \theta}+b_{\theta}=\mu \ddot{u}_{\theta}, \\
& \frac{\partial \sigma_{r z}}{\partial r}+\frac{1}{r} \frac{\partial \sigma_{\theta z}}{\partial \theta}+\frac{\partial \sigma_{z}}{\partial z}+\frac{1}{r} \sigma_{r z}+b_{z}=\mu \ddot{u}_{z},
\end{aligned}
$$

where $\mu$ is the mass density of the material, $b_{r}, b_{\theta}, b_{z}$ are the body forces per unit volum, $\ddot{u}_{r}, \ddot{u}_{\theta}, \ddot{u}_{z}$ the acceleration. The strains can be written, in cylindrical coordinate, as:

$$
\begin{gathered}
\varepsilon_{r}=\frac{\partial u_{r}}{\partial r}, \varepsilon_{\theta}=\frac{1}{r} \frac{\partial u_{\theta}}{\partial \theta}+\frac{u_{r}}{r}, \varepsilon_{z}=\frac{\partial u_{z}}{\partial z} \\
\varepsilon_{r \theta}=\frac{1}{2}\left(\frac{1}{r} \frac{\partial u_{r}}{\partial \theta}+\frac{\partial u_{\theta}}{\partial r}-\frac{u_{\theta}}{r}\right), \varepsilon_{r z}=\frac{1}{2}\left(\frac{\partial u_{r}}{\partial z}+\frac{\partial u_{z}}{\partial r}\right), \varepsilon_{\theta z}=\frac{1}{2}\left(\frac{\partial u_{\theta}}{\partial z}+\frac{1}{r} \frac{\partial u_{z}}{\partial \theta}\right)
\end{gathered}
$$

where $\varepsilon_{r}, \varepsilon_{\theta}, \varepsilon_{z}, \varepsilon_{r \theta}, \varepsilon_{r z}, \varepsilon_{\theta z}$ are the strains components, $\lambda$ and $\mathrm{G}$ are the Lamè constants.

The stresses can be obtained using the generalized Hooke law:

$$
\begin{gathered}
\sigma_{r}=\lambda\left(\frac{\partial u_{r}}{\partial r}+\frac{1}{r} \frac{\partial u_{\theta}}{\partial \theta}+\frac{u_{r}}{r}+\frac{\partial u_{z}}{\partial z}\right)+2 G \frac{\partial u_{r}}{\partial r} \\
\sigma_{\theta}=\lambda\left(\frac{\partial u_{r}}{\partial r}+\frac{1}{r} \frac{\partial u_{\theta}}{\partial \theta}+\frac{u_{r}}{r}+\frac{\partial u_{z}}{\partial z}\right)+2 G\left(\frac{1}{r} \frac{\partial u_{\theta}}{\partial \theta}+\frac{\partial u_{r}}{\partial r}\right) \\
\sigma_{z}=\lambda\left(\frac{\partial u_{r}}{\partial r}+\frac{1}{r} \frac{\partial u_{\theta}}{\partial \theta}+\frac{u_{r}}{r}+\frac{\partial u_{z}}{\partial z}\right)+2 G \frac{\partial u_{z}}{\partial z} \\
\sigma_{r \theta}=G\left(\frac{1}{r} \frac{\partial u_{r}}{\partial \theta}+\frac{\partial u_{\theta}}{\partial r}-\frac{u_{\theta}}{r}\right) \\
\sigma_{r z}=G\left(\frac{\partial u_{r}}{\partial z}+\frac{\partial u_{z}}{\partial r}\right) \\
\sigma_{\theta z}=G\left(\frac{\partial u_{\theta}}{\partial z}+\frac{1}{2 r} \frac{\partial u_{z}}{\partial \theta}\right)
\end{gathered}
$$

If we denote the cubic dilatation with:

$$
I_{1 \varepsilon}=\varepsilon_{x}+\varepsilon_{y}+\varepsilon_{z}=\frac{\partial u_{r}}{\partial r}+\frac{1}{r} \frac{\partial u_{\theta}}{\partial \theta}+\frac{u_{r}}{r}+\frac{\partial u_{z}}{\partial z}
$$

the motion Equation (1) can be written:

$$
\begin{gathered}
G\left(\nabla^{2} u_{r}-\frac{u_{r}}{r^{2}}-\frac{2}{r^{2}} \frac{\partial u_{\theta}}{\partial \theta}\right)+(\lambda+G) \frac{\partial I_{1 \varepsilon}}{\partial \varepsilon}+b_{r}=\mu \ddot{u}_{r} \\
G\left(\nabla^{2} u_{\theta}-\frac{u_{\theta}}{r^{2}}+\frac{2}{r^{2}} \frac{\partial u_{r}}{\partial \theta}\right)+(\lambda+G) \frac{1}{r} \frac{\partial I_{1 \varepsilon}}{\partial \theta}+b_{\theta}=\mu \ddot{u}_{\theta} \\
G \nabla^{2} u_{\theta}+(\lambda+G) \frac{\partial I_{1 \varepsilon}}{\partial z}+b_{z}=\mu \ddot{u}_{z}
\end{gathered}
$$

or:

$$
G \nabla^{2} u+(\lambda+G) \nabla(\nabla u)=\mu \frac{\partial u}{\partial t^{2}} .
$$

The equations represents a system of three, second-order partial differential equations. The equations are, generally, complex, with many terms. A verified and widely used method of solving is FEM.

\section{Use of FEM for the Study of a Hollow Cylinder}

Consider a hollow cylinder as a linear elastic solid. The hollow cylinder is made by two identical parts, separated with a liaison plan (Figure 1). At the same time, if we divide 
the cylinder in two, using a transversal plan xy, these two parts are identical. Here, we have multiple symmetries.
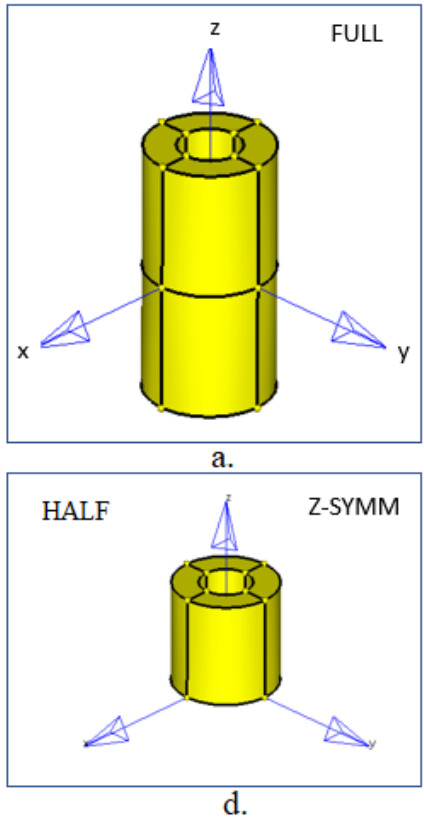

d.
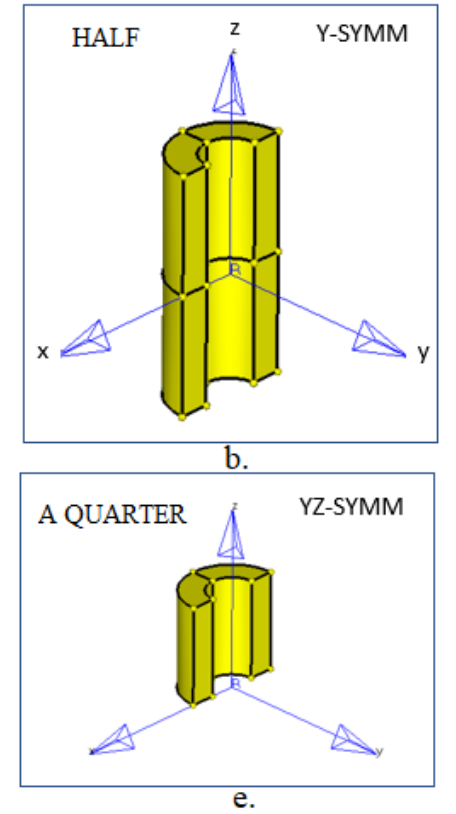
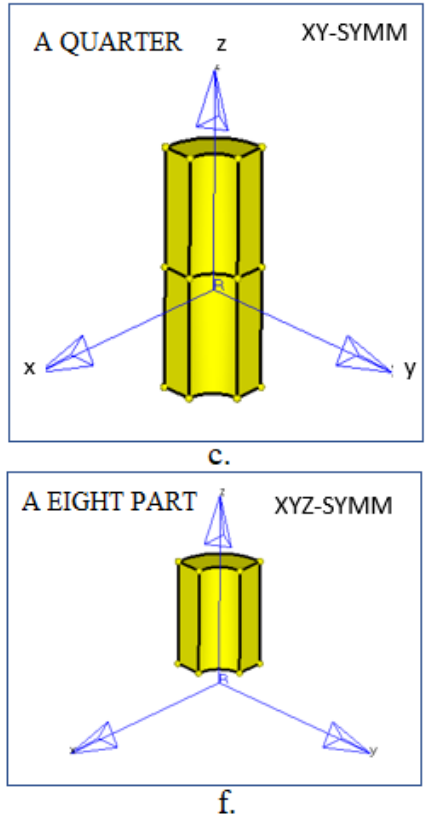

Figure 1. The hollow cylinder considered composed of different identical parts. (a) Full cylinder; $(\mathbf{b}, \mathbf{d})$ half cylinder; (c,e) quarter cylinder; (f) eight parts of a cylinder.

The motion equations of the free undamped vibrations, using FEM, can be written under the form [31-34]:

$$
[m]\{\ddot{x}\}+[k]\{x\}=0
$$

where:

- $\quad[m]$ is the mass matrix;

- $\quad[k]$ the stiffness matrix.

Element type used in analyzes: HEX8 - first order hexahedral element with 8 corner nodes, each node having 3 degrees of nodal freedom.

Let us consider now the problem of determining the eigenvalues for a hollow cylinder represented in Figure 1. This system, denoted with $(S)$, is composed of two identical subsystems $\left(S_{1}\right)$-half cylinder. Using the classical methods of mechanics [35-38] is possible to write the equations of the free non-damped vibrations for the whole structure as:

$$
\left[\begin{array}{cccc}
m_{a} & 0 & m_{a b} & m_{a b} \\
0 & m_{a} & m_{a b} & m_{a b} \\
m_{a b} & m_{a b} & m_{b} & 0 \\
m_{a b} & m_{a b} & 0 & m_{b}
\end{array}\right]\left\{\begin{array}{c}
\ddot{x}_{s} \\
\ddot{x}_{d} \\
\ddot{x}_{s d} \\
\ddot{x}_{d s}
\end{array}\right\}+\left[\begin{array}{cccc}
k_{a} & 0 & k_{a b} & k_{a b} \\
0 & k_{a} & k_{a b} & k_{a b} \\
k_{a b} & k_{a b} & k_{b} & 0 \\
k_{a b} & k_{a b} & 0 & k_{b}
\end{array}\right]\left\{\begin{array}{c}
x_{s} \\
x_{d} \\
x_{s d} \\
x_{d s}
\end{array}\right\}=0
$$

$m_{a}, m_{b}, k_{a}, k_{b}$ are symmetrical square matrices; $m_{a}$ is the mass matrix for a half cylinder, $m_{b}$ the mass matrix for the nodes of the common part (the nodes being in the common plan of the identical parts), $k_{a}$ the stiffness matrix of the half-cylinder, $k_{b}$ the stiffness matrix of the system common part (assuring the elastic liaison between the nodes being in the common plan and the other nodes of the identical parts), $m_{a b}$ and $k_{a b}$ being the mass, respectively, stiffness matrix that describes the liaison between the two identical parts via the common plan.

For half a structure, the equations become:

$$
\left[m_{a}\right]\left\{x_{s}\right\}+\left[k_{a}\right]\left\{x_{s}\right\}=0
$$


for the left part and

$$
\left[m_{a}\right]\left\{x_{d}\right\}+\left[k_{a}\right]\left\{x_{d}\right\}=0
$$

for the right part. These two equations are identical and have the same eigenvalues and eigenvectors. By numerical calculation, using the method of finite elements [11,12,14], the following property has been established:

Property 1: The eigenvalues for system (9) are eigenvalues for system (8) as well. That means the solutions of algebraic equations

$$
\operatorname{det}\left(\left[k_{a}\right]-\omega^{2}\left[m_{a}\right]\right)=0\left(\text { or }\left|\left[k_{a}\right]-\omega^{2}\left[m_{a}\right]\right|=0\right)
$$

are also solutions of the algebraic equation

$$
\operatorname{det}\left(\left[\begin{array}{cccc}
m_{a} & 0 & m_{a b} & m_{a b} \\
0 & m_{a} & m_{a b} & m_{a b} \\
m_{a b} & m_{a b} & m_{b} & 0 \\
m_{a b} & m_{a b} & 0 & m_{b}
\end{array}\right]-\omega^{2}\left[\begin{array}{cccc}
k_{a} & 0 & k_{a b} & k_{a b} \\
0 & k_{a} & k_{a b} & k_{a b} \\
k_{a b} & k_{a b} & k_{b} & 0 \\
k_{a b} & k_{a b} & 0 & k_{b}
\end{array}\right]\right)=0
$$

or

$$
\left|\begin{array}{cccc}
k_{a}-\omega^{2} m_{a} & 0 & k_{a b}-\omega^{2} m_{a b} & k_{a b}-\omega^{2} m_{a b} \\
0 & k_{a}-\omega^{2} m_{a} & k_{a b}-\omega^{2} m_{a b} & k_{a b}-\omega^{2} m_{a b} \\
k_{a b}-\omega^{2} m_{a b} & k_{a b}-\omega^{2} m_{a b} & k_{b}-\omega^{2} m_{b} & 0 \\
k_{a b}-\omega^{2} m_{a b} & k_{a b}-\omega^{2} m_{a b} & 0 & k_{b}-\omega^{2} m_{b}
\end{array}\right|=0
$$

We could write:

$$
\left|\left[k_{a}\right]-\omega^{2}\left[m_{a}\right]\right|=0 \Rightarrow
$$

that implies the polynomial in $\omega^{2}$ from Equation (11) is divided by the polynomial in $\omega^{2}$ from Equation (10). In following is presented the Proposition, proved in [39], that assures the validity of the Property 1 .

Proposition: Consider the square polynomial matrices with complex coefficients, of size $\mathrm{n}$, noted $A, B, C, L, Z=O_{n}$ and matrix

$$
M=\left(\begin{array}{ccc}
A & Z & B \\
Z & A & B \\
L & L & C
\end{array}\right)
$$

then $\operatorname{det}(M)$ is $\operatorname{dividable~by~} \operatorname{det}(A)$.

\section{Eigenvalues and Eigenmodes}

In a line of Table 1 are presented the common eigenvalues, property presented before. The eigenvalues of a part of the cylinder are eigenvalues for the whole cylinder.

The eigenmodes are represented in Table 1. The properties presented before are sustained by the graphical representation on this table. We have two kinds of symmetry. If we look to the last line of the table we can see that the eigenfrequency $22,043 \mathrm{~Hz}$ is obtained for six different type of element. This thing is happening for all the symmetry that can be built for the cylinder.

The paper aims to show that considering the symmetries that occur within a structure can lead to the identification of properties that allow ease of calculation. For example, in the case presented in the paper, that of a cylinder, the consideration of some substructures of the cylinder allows to reduce the size of the problem of calculating the eigenvalues and eigenmodes. Thus, if we consider for example a quarter of the cylinder, all eigenvalues calculated for this substructure are found between the eigenvalues of the entire cylinder. In this, using the existing symmetries, calculations can be made on substructures reduced 
in size. The calculation for these is easier and in this way a number of eigenvalues can be determined for the whole structure. In this way, knowing these eigenvalues, the size of the initial system and, consequently, the necessary computational effort can be significantly reduced. In general, the numerical solution of some problems leads to an exponential explosion of the necessary computational effort [39]. FEM is only an analysis tool, considered appropriate by the authors for analyzing the problem.

Table 1. The eigenmodes of the studied parts (Hz).

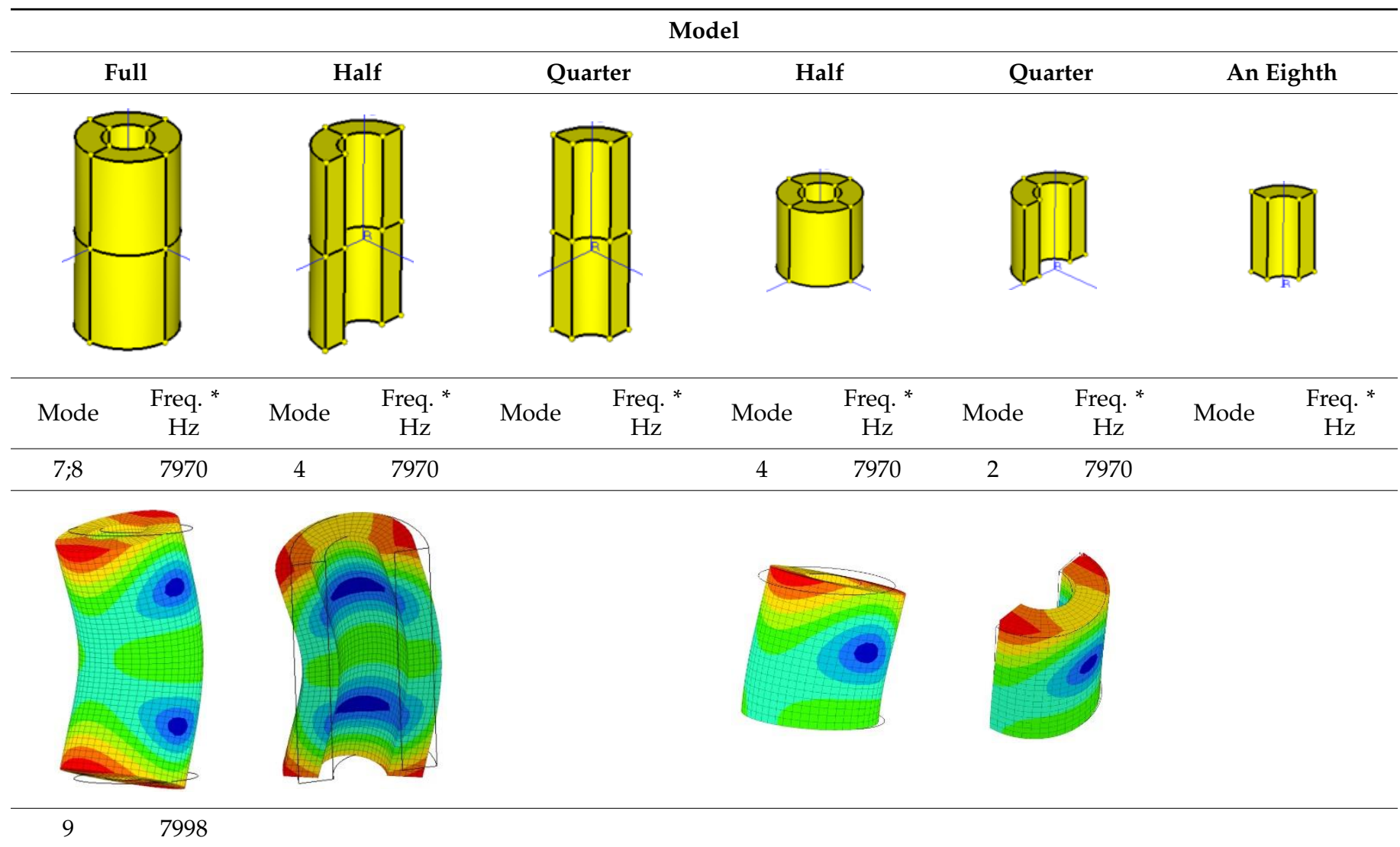

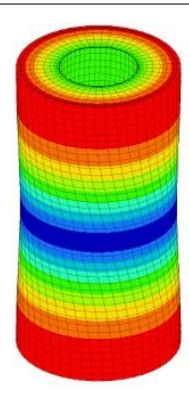

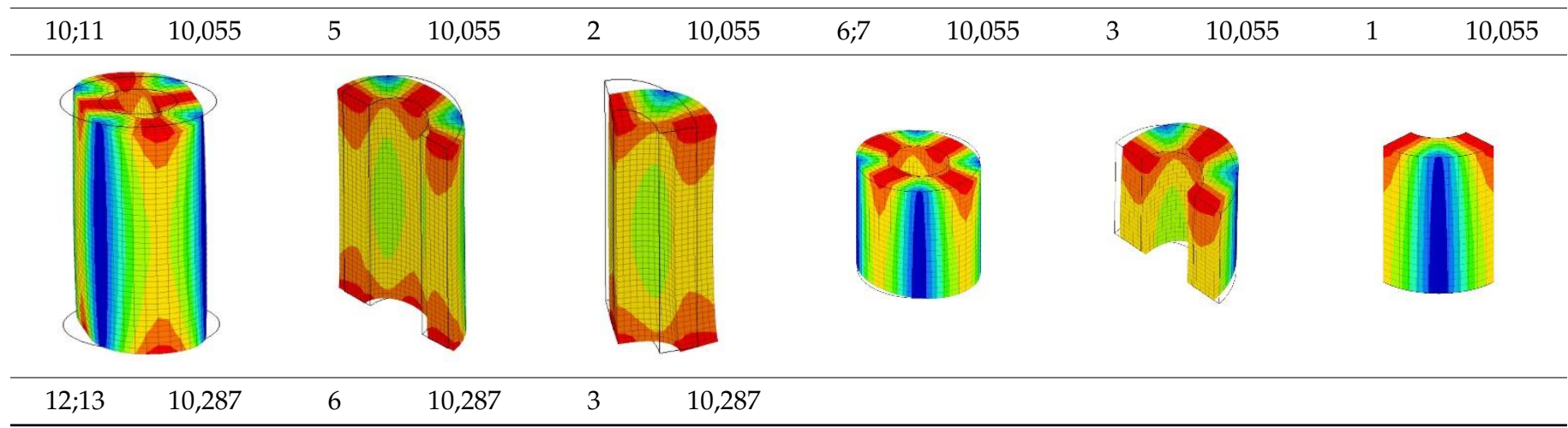


Table 1. Cont

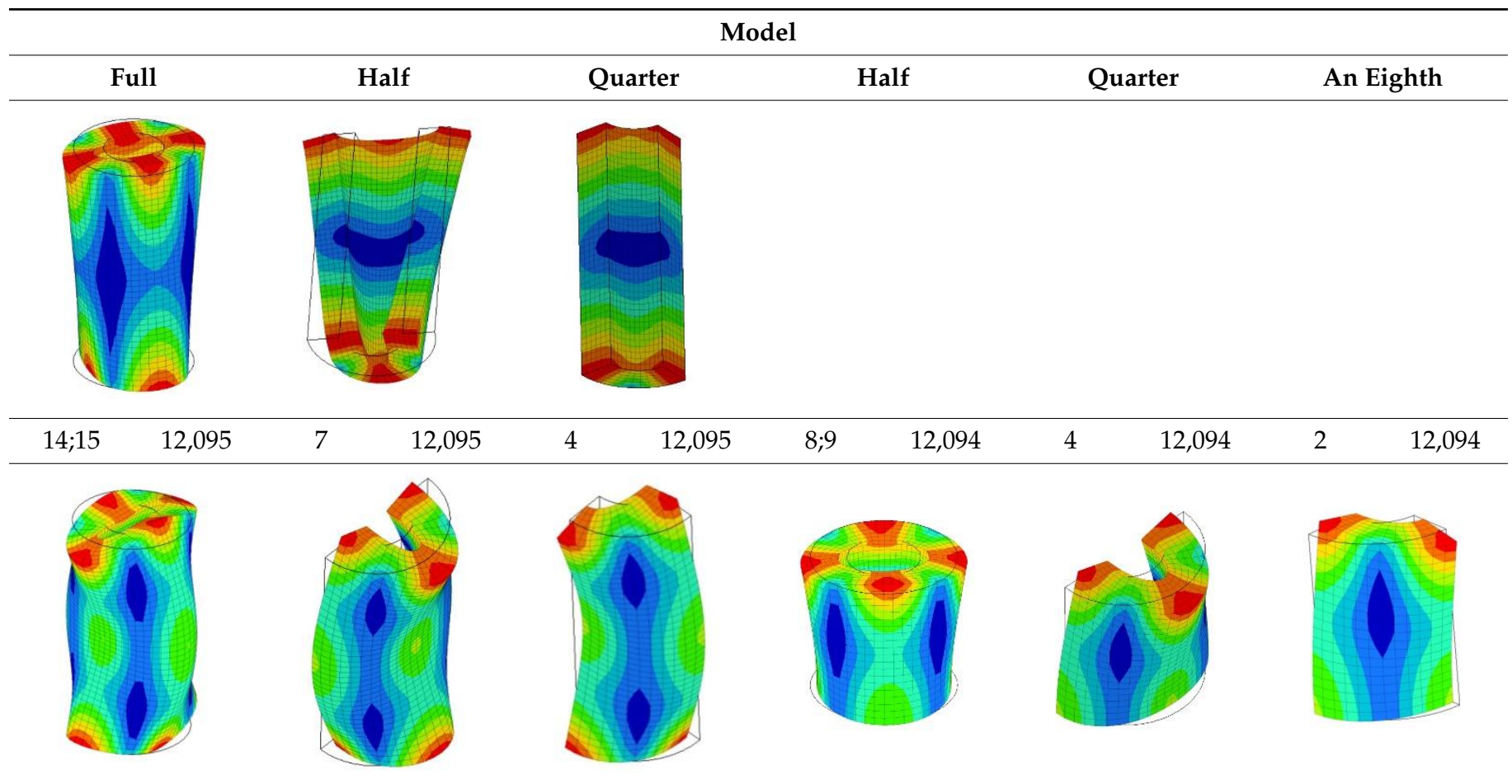

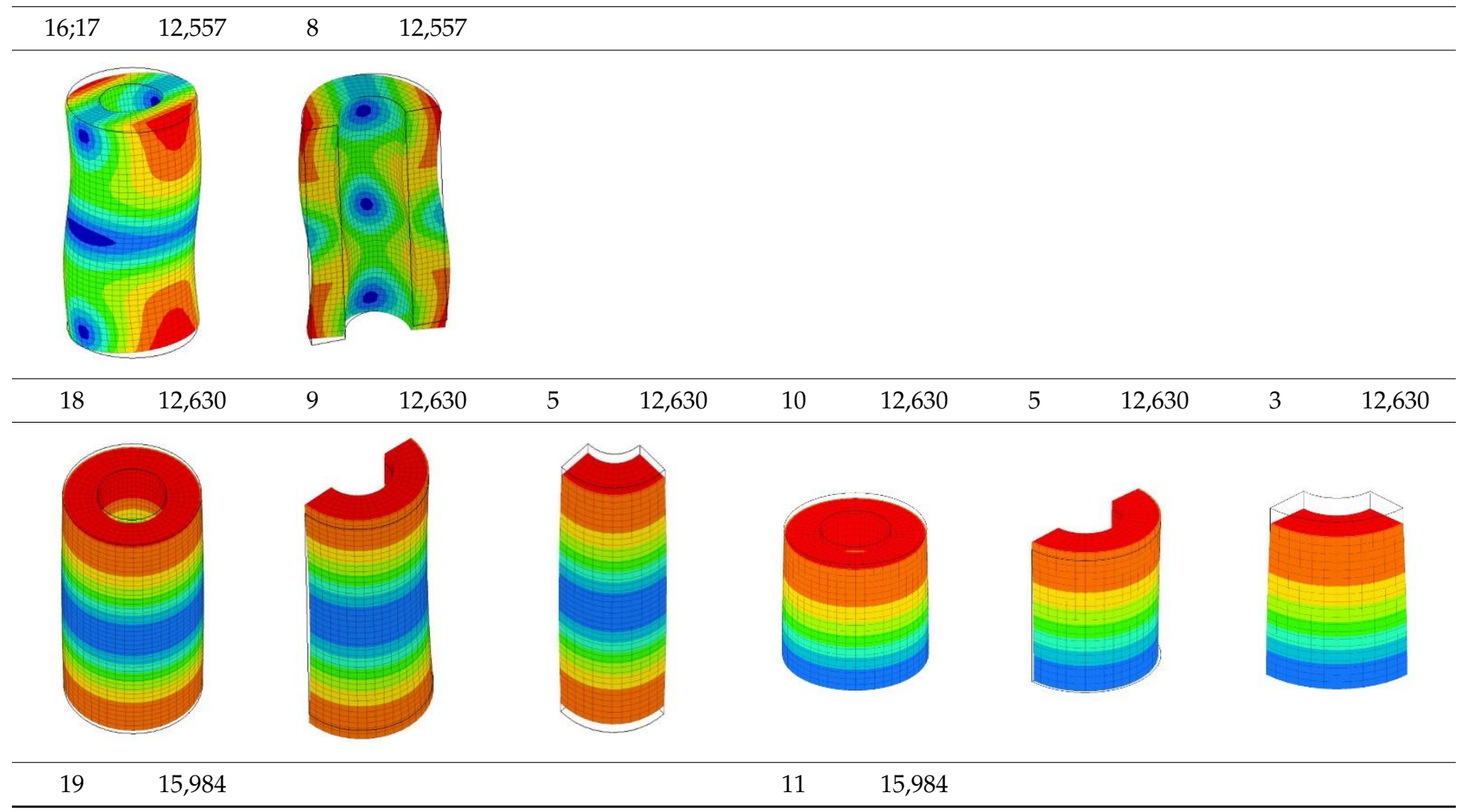


Table 1. Cont

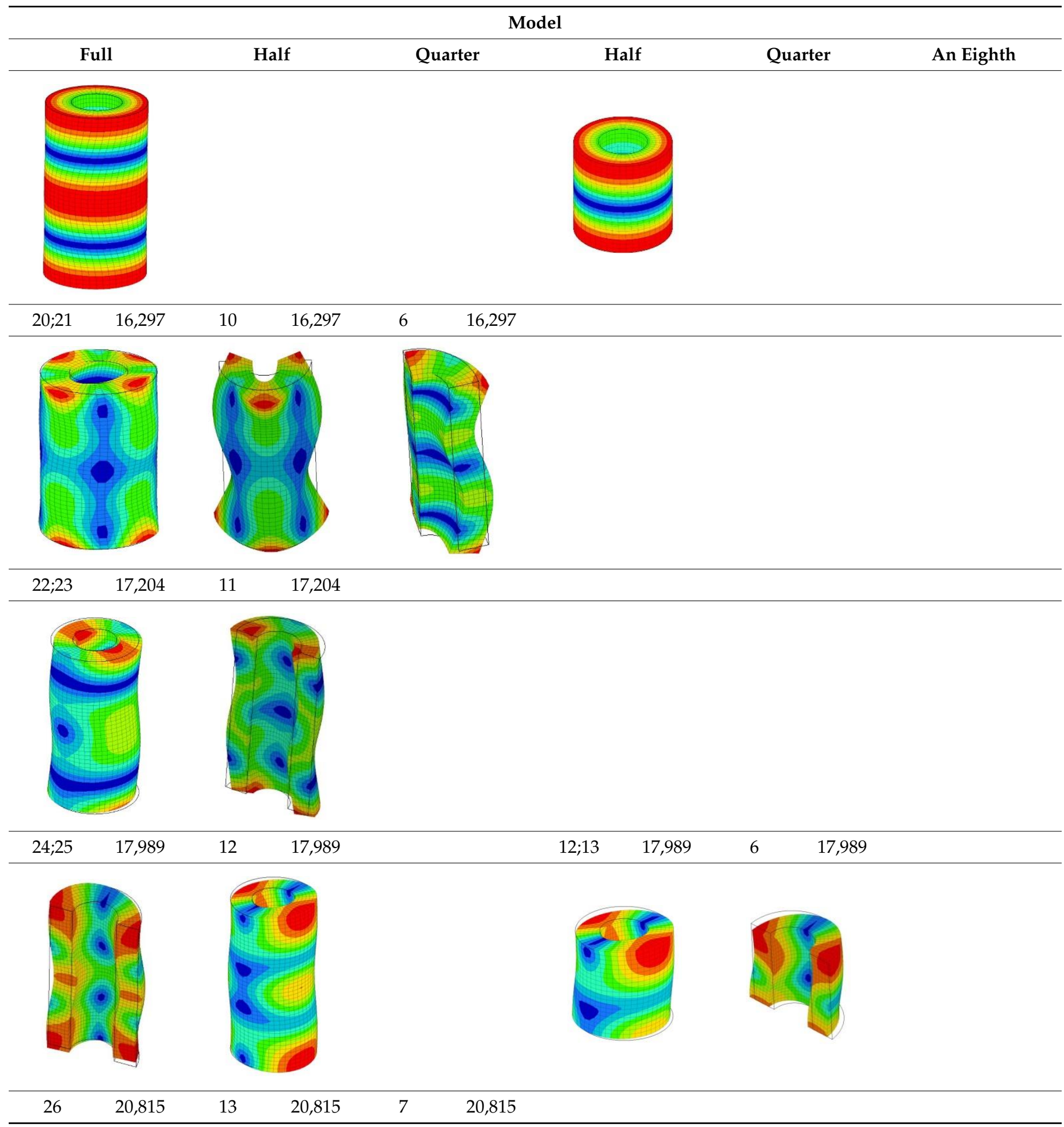


Table 1. Cont.

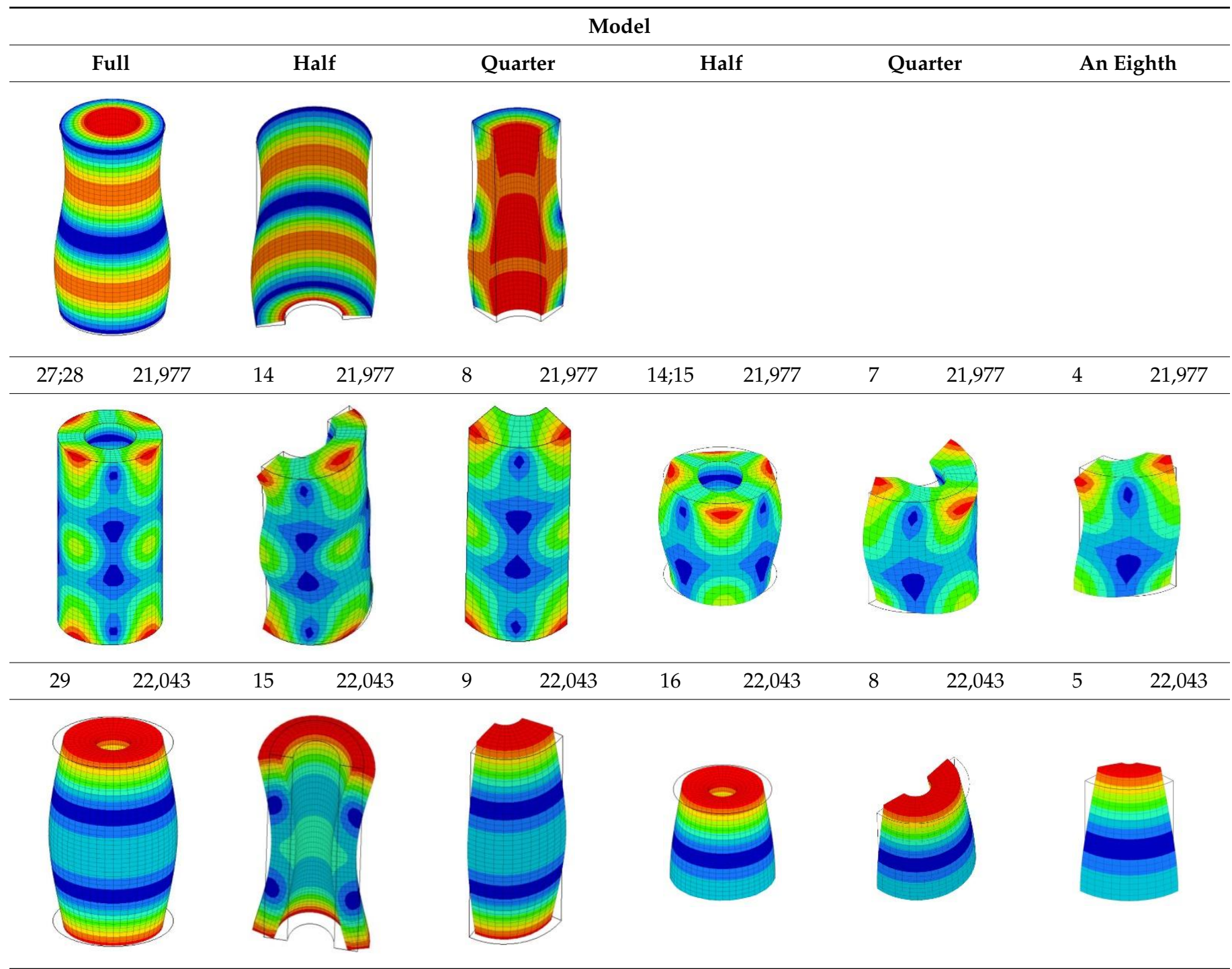

${ }^{*}$ Freq.-represents the calculated frequency in Hz.

\section{Discussion and Conclusions}

The main field of applicability of these properties is in the field of civil engineering but, just as well, there are applications in all branches of engineering. Experimental measurement in order to verify such type of model are presented in [38].

The issue addressed in this paper has been relatively little studied; however, some papers in the field exist, and the importance of repetitive elements (parts) or symmetries in performing stress and strain calculations has been observed for a long time. In this paper, we have proved that for a mechanical linear elastic system consisting of two identical subsystems, the eigenfrequencies of repetitive subsystems are eigenvalues of the entire structure as well. At the same time, the eigenmodes present certain symmetries. These properties allow the simplification of the vibration calculus for this type of structure. We can first determine the eigenvalues of the symmetric structure, then these eigenvalues can be eliminated from the motion equations of motion for the whole structure. Thus, the size of the problem decreases and allows a reduced cost in computation. The symmetries presented by eigenvectors allow a quantitative understanding of vibration phenomena in the system. The modes of the whole structure can be obtained from the modes of its one substructure. The properties can be useful for an experimental procedure. It is necessary to 
perform the measurement only for a half body, using the described properties is possible to know the modes for the entire structure.

If we take into account the properties demonstrated in the paper, it is enough to calculate eigenfrequencies and eigenmodes only for half of the structure, or a quarter of the structure. Appropriate boundary conditions must be imposed in the symmetry plane. The eigenpulsations of such a structure are also the eigenpulsations of the whole structure, and therefore the size of the system can be reduced using classical methods from algebra. The number of degrees of freedom decreases by almost half, and thus the calculation necessary for the determination of its eigenpulsations and its eigenmodes is significantly reduced, and as a result, the cost of the calculation and design operations of such a structure decreases.

The eigenmodes of the whole structure are obtained by the natural extension of the symmetric modes obtained for a substructure.

At the experimental level, if eigenfrequency measurements are made only for half of the structure, they are also the eigenfrequency for the whole structure.

Author Contributions: Formal analysis, C.I.; investigation, C.I.; methodology, C.I.; supervision, C.I.; validation, C.I.; visualization, C.I.; conceptualization, S.V.; funding acquisition, S.V.; Investigation, S.V.; methodology, S.V.; software, S.V.; supervision, S.V.; visualization, S.V.; writing—original draft, S.V.; writing —review and editing, S.V.; conceptualization, M.M.; Formal analysis, M.M.; methodology, M.M.; supervision, M.M.; validation, M.M.; data curation, A.T.; Formal analysis, A.T.; project administration, A.T.; resources, A.T.; software, A.T. All authors have read and agreed to the published version of the manuscript.

Funding: The APC was funded by the Transilvania University of Brasov.

Conflicts of Interest: The authors declare no conflict of interest.

\section{References}

1. Wang, D.; Zhou, C.; Rong, J. Free and forced vibration of repetitive structure. Int. J. Solids Struct. 2003, 40, 5477-5494. [CrossRef]

2. Cai, C.; Wu, F. On the Vibration of RotationalPeriodic Structures. In Acta Scientiarum Naturalium Universitatis Sunyatseni; Sun Yat-sen University: Guangzhou, China, 1983; Volume 22, p. 109.

3. Cai, C.; Cheung, Y.; Chan, H. Uncoupling of dynamic equations for periodic structures. J. Sound Vib. 1990, 139, $253-2630$. [CrossRef]

4. Chan, H.; Cai, C.; Cheung, Y. Exact Analysis of Structures with Periodicity Using U-Transformation; World Scientific Publication: Singapore, 1998.

5. Evensen, D.A. Vibration Analysis of Multi-symmetric structures. AIAA J. 1976, 14, 446-453. [CrossRef]

6. Meirovitch, L. Elements of Vibration Analysis, 2nd ed.; McGraww-Hill: New York, NY, USA, 1970.

7. Wang, D.; Wang, C.C. Natural vibration of repetitive structures. Chin. J. Mech. 2000, 16, 85-95. [CrossRef]

8. Zhong, $W$. The eigen-value problem of the chain of identical substructures and the expansion method solution lasted on the eigen-vectors. Acta Mech. Sin. 1991, 23, 72-81.

9. Brillouin, L. Wave Propagation in Periodic Structures; Dover Publications: New York, NY, USA, 1946.

10. Mead, D.J. A general theory of harmonic wave propagation in linear periodic systems with multiple coupling. J. Sound Vib. 1973, 27, 235-260. [CrossRef]

11. Mead, D.J. Wave propagation and naturalmodes in periodic systems. I. Mono-coupled systems. J. Sound Vib. 1975, 40, 1-18. [CrossRef]

12. Mead, D.J. Wave propagation and naturalmodes in periodic systems. II. Multi-coupled systems, with and without damping. J. Sound Vib. 1975, 40, 19-39. [CrossRef]

13. Mead, D.J.; Bansal, A.S. Mono-coupled periodic systems with a single disorder: Free wave propagation. J. Sound Vib. 1978, 61, 481-496. [CrossRef]

14. Gry, L.; Gontier, C. Dynamic modelling of railway track: A periodic model on a generalized beam formulation. J. Sound Vib. 1997, 199, 531-558. [CrossRef]

15. Zhong, W.H.; Williams, F.W. On the direct solution of wave propagation for repetitive structures. J. Sound Vib. 1995, 181, 485-501. [CrossRef]

16. Vlase, S.; Teodorescu-Drăghicescu, H.; Scutaru, L.M. On the Eigenvalues of the Elastic Systems with Some Symmetries. In Proceedings of the 6th International DAAAM Baltic Conference Industrial Engineering, Tallinn, Estonia, 24-26 April 2008.

17. Vlase, S.; Paun, M. Vibration analysis of a mechanical system consisting of two identical parts. R. J. Tech. Sci. Appl. Mech. 2015, 60, 216-230. 
18. Kaveh, A.; Hamedani, K.B.; Joudaki, A.; Kamalinejad, M. Optimal analysis for optimal design of cyclic symmetric structures subject to frequency constraints. Structures 2021, 33, 3122-3136. [CrossRef]

19. Vlase, S.; Marin, M.; Scutaru, M.L.; Pruncu, C. Vibration Response of a Concrete Structure with Repetitive Parts Used in Civil Engineering. Mathematics 2021, 9, 490. [CrossRef]

20. Vlase, S.; Teodorescu, P.P. Elasto-Dynamics of a Solid with a general "Rigid" Motion using FEM Model. Part I. Theoretical Approach. Rom. J. Phys. 2013, 58, 872-881.

21. Zhang, J.; Reynders, E.; De Roeck, G.; Lombaert, G. Model updating of periodic structures based on free wave characteristics. J. Sound Vib. 2019, 442, 281-307. [CrossRef]

22. $\mathrm{Wu}, \mathrm{L} . J . ;$ Song, H.W. Band gap analysis of periodic structures based on cell experimental frequency response functions (FRFs). Acta Mech. Sin. 2019, 35, 156-173. [CrossRef]

23. Ashari, A.K.; Stephen, N.G. On wave propagation in repetitive structures: Two forms of transfer matrix. J. Sound Vib. 2019, 439, 99-112. [CrossRef]

24. Kaveh, A.; Joudaki, A. Matrix Analysis of Repetitive Circulant Structures: New-block and Near Block Matrices. Period. Polytech Civ. Eng. 2019, 63, 687-694. [CrossRef]

25. Rallu, A.; Hans, S.; Boutin, C. Asymptotic analysis of high-frequency modulation in periodic systems. Analytical study of discrete and continuous structures. J. Mech. Phys. Solids 2018, 117, 123-156. [CrossRef]

26. Kaveh, A.; Zolghadr, A. Optimal design of cyclically symmetric trusses with frequency constraints using cyclical parthenogenesis algorithm. Adv. Struct. Eng. 2018, 21,739-755. [CrossRef]

27. Li, L.Y.; Cheng, B.W.; Zhang, Y.; Qin, H. Study of a smart platform based on backstepping control method. Earthq. Eng. Eng. Vib. 2017, 16, 599-608. [CrossRef]

28. Viola, E.; Alessandro, M. Exact Analysis of Wave Motions in Rods and Hollow Cylinders. In Mechanical Vibration: Where Do We Stand? Elishakoff, I., Ed.; Springer: Berlin/Heidelberg, Germany; Wien: New York, NY, USA, 2006.

29. Viola, E.; Alessandro, M.; Bartoli, I. Numerical Evaluation of Semi-analytical Finite Element (SAFE) Method for Plates, Rods and Hollow Cylinders. In Mechanical Vibration: Where Do We Stand? Elishakoff, I., Ed.; Spinger: Berlin/Heidelberg, Germany; Wien: New York, NY, USA, 2006.

30. Viola, E.; Alessandro, M.; Bartoli, I. Semi-analytical Formulation for Guided Wave Propagation. In Mechanical Vibration: Where Do We Stand? Elishakoff, I., Ed.; Spinger: Berlin/Heidelberg, Germany; Wien: New York, NY, USA, 2006.

31. Haris, C.M.; Crede, C.E. Shock and Vibration Handbook, 6th ed.; McGraw-Hill: New York, NY, USA, 2009.

32. Horn, R.A.; Johnson, C.R. Matrix Analysis; Cambridge University Press: Cambridge, UK, 1985.

33. Vlase, S. Elimination of Lagrangian Multipliers. Mech. Res. Commun. 1987, 14, 17-22. [CrossRef]

34. Vlase, S. A Method of Eliminating Lagrangean Multipliers from the Equation of Motion of Interconnected Mechanical System. ASME Transaction. J. Appl. Mech. 1987, 54, 235-236. [CrossRef]

35. Scutaru, M.L.; Vlase, S. Some Properties of Motion Equations Describing the Nonlinear Dynamical Response of a Multibody System with Flexible Elements. J. Appl. Math. 2012. [CrossRef]

36. Vlase, S.; Teodorescu, P.P.; Itu, C.; Scutaru, M.L. Elasto-Dynamics of a Solid with a General “Rigid” Motion Using FEM Model. Part II. Analysis of a Double Cardan Joint. Rom. J. Phys. 2013, 58, 882-892.

37. Vlase, S.; Dănășel, C.; Scutaru, M.L.; Mihălcică, M. Finite Element Analysis of a Two-Dimensional Linear Elastic Systems with a plane "Rigid Motion". Rom. J. Phys. 2014, 59, 476-487.

38. Vasile, O.; Vlase, S.; Nastac, C.; Scutaru, M.L. Experimental Analysis of a Mechanical System Composed by Two Identical Parts. Acta Technica Napocensis, Series-Applied Mathematics. Mech. Eng. 2018, 61, 333-338.

39. Bakhvalov, N.S. Numerical Methods: Analysis, Algebra, Ordinary Differential Equations; MIR Publisher: Moscow, Russia, 1977. 